\title{
IDENTIFICAÇÃO DO RISCO DE CARDIOPATIA ATRAVÉS DO ESTUDO COMBINADO DE CIRCUNFERÊNCIAS CORPORAIS
}

\author{
Vivianne Correia dos Santos MORAES*, João Romário Gomes DA SILVA, Fábio Luiz Fully TEIXEIRA, Bruno \\ Correia Ulisses SOBREIRA, Juçara Gonçalves Lima BEDIM \& Marcus Lima BEDIM
}

Universidade Iguaçu (UNIG), campus $V$, Itaperuna, Rio de Janeiro, Brasil.

* Autora para correspondência: vivineuro@ hotmail.com

http://dx.doi.org/10.18571/acbm.096

\section{RESUMO}

De acordo com o modelo geométrico desenvolvido pelo New York Obesity Research Center (NYORC), o corpo humano é assemelhado a um cilindro, cuja altura e comprimento são proporcionais. Destacam- se, assim, os perímetros braquial, da cintura, do quadril, da coxa e da panturrilha como marcadores antropométricos de risco para doenças cardiovasculares. O objetivo foi descrever o valor preditivo da correlação entre as circunferências corporais e marcadores antropométricos de risco para doenças cardiovasculares. Trata-se de estudo transversal comparativo de abordagem quantitativa e qualitativa, realizado com 60 (sessenta) pacientes, cardiopatas ou não, durante atendimento nos ambulatórios do Hospital São José Avaí (HSJA), em Itaperuna/RJ. A organização amostral ocorreu em dois grupos: o dos cardiopatas (G1) e o dos não cardiopatas (G2). Foram elaboradas tabelas descritivas, utilizando-se frequência, média e desvio padrão. Na análise das frequências, o teste qui-quadrado de Pearson preditivo, o nível de significância estatística foi fixado em $\mathrm{p}<0,05$. A média geral de idade foi de $47( \pm 13,34)$ anos. G1 exibiu médias superiores a $\mathrm{G} 2$, no que tange aos valores antropométricos, ao IMC e às médias das circunferências corporais. $\mathrm{O}$ coeficiente de correlação de Pearson demonstrou correlação fortemente significativa entre os índices de circunferência e altura/volume $\left(\mathrm{V}=\pi \cdot(\mathrm{C} / 2 \pi)^{2} . \mathrm{H}\right)$, demonstrado através de matrizes quadradas. As circunferências da cintura e do quadril $(\rho=0,998$ e $\rho=0,999)$ apresentaram proporções semelhantes nos dois grupos. $\mathrm{O}$ valor preditivo das circunferências corporais para doenças cardiovasculares é necessário tanto para a prevenção quanto para o tratamento das cardiopatias.

Palavras-chave: Cardiopatias; Obesidade; Fatores de Risco; Índice de Massa Corpórea; Infarto do Miocárdio.

\section{ABSTRACT}

According to the geometric model developed by the New York Obesity Research Center (NYORC), the human body is like a cylinder. Thus, the brachial, waist, hip, thigh and calf girth appears advantageous as anthropometric markers for cardiovascular disease risk. The purpose of this study was to describe the predictive value of the correlation between body circumferences and anthropometric markers for heart disease risk. Cross-sectional comparative study with 60 (sixty) patients, with or without heart disease. Data were collected on patients seen at outpatients clinics in Hospital São José do Avaí, in Itaperuna, Rio de Janeiro, Brazil. The study included two groups of patients: group 1 (G1), those who have heart disease, and, group 2 (G2), those without heart disease. Descriptive statistics were conducted, including the mean and standard derivation. On the bivariate statistical analysis, it was used the Pearson chi-square test and the significance level was set on 0,05. The average age was $47( \pm 13,34)$ years. The values of body circumferences and body mass index 
(BMI) in G1 were significantly higher than those in G2. We tested the correlation between body circumferences and height/size ratio $\left(\mathrm{V}=\pi \cdot(\mathrm{C} / 2 \pi)^{2} . \mathrm{H}\right)$. The relationship between waist and hip $(\rho=$ 0,998 e $\rho=0,999$ ) showed similar results in both groups. It was concluded that prediction equations using circumferences (together with other anthropometric measures) predict incident cardiovascular events.

Keywords: Cardiovascular Diseases; Obesity; Risk Factors; Body Mass Index; Myocardial Infarction.

\section{Introdução}

De acordo com os Parâmetros Curriculares Nacionais do curso de graduação em Medicina (BRASIL, 2001), o médico deverá "promover estilos de vida saudáveis... atuando como agente social na transformação". Diante desta perspectiva, é oportuno que o profissional médico opte por intervir, na matriz da patologia, ou seja, na interpenetração continua entre prevenção e tratamento Destarte, a ênfase no combate à obesidade é fundamental.

Inicialmente, verifica-se que a obesidade é uma doença crônica, equacionada por fatores ambientais e genéticos, precursores pró-inflamatórios nos adipócitos, cujos alvos são o sistema metabólico e o sistema cardiovascular (FARIA et al., 2013).

Ante esse panorama, como bem pontuam alguns estudos (HEITMANN, 2009; JUNG et al., 2013), a obesidade confere um aumento da suscetibilidade para o desenvolvimento de doenças cardiovasculares. Assim, há um forte valor entre o índice de Massa Corpórea (IMC) e o Infarto Agudo do Miocárdio (IAM), tendo como recorrência o desfecho de mortalidade.

Notadamente, assinala-se que a obesidade apresenta profundas ressonâncias, ainda, no remodelamento cardíaco (ABEL; LITWIN; SWEENEY, 2008), no desenvolvimento da síndrome metabólica, das doenças ateroscleróticas, da Hipertensão Arterial Sistêmica (HAS), do diabetes e das dislipidemias.

Em especial, no que tange ao remodelamento cardíaco, o Framingham Heart Study concluiu que a obesidade central é um marcador de risco (HSIEH; YOSHINAGA, 1995). Além disso, ela provoca mudanças e hipertrofia das câmaras cardíacas, bem como é precursora de formas subclínicas: sistólicas e diastólicas.

A partir dessa premissa, os autores deste estudo estabeleceram o modelo geométrico desenvolvido pelo New York Obesity Research Center (NYORC), denominado "Modelo das Circunferências Corporais", Fase I (HEYMSFIELD et al., 2008), como arcabouço teórico metodológico ao encaminhamento da investigação. De fato, a Fase I da pesquisa original desenvolveu uma lógica matemática associativa de medidas corporais às tendências fisiopatológicas cardíacas. Assim, assinala-se que o corpo contém cinco circunferências obtidas a partir dos perímetros braquial, da cintura, do quadril, da coxa e da panturrilha. $\mathrm{O}$ estudo preconiza que, quando a equivalência corporal é perdida, há sérias repercussões sistêmicas, o que desencadeia processos patológicos, cujo principal expoente é a falha cardíaca.

Para fins didáticos, optou-se por considerar o volume corporal equivalente ao peso corporal, pressupondo-se, assim, que, os dois são medidas aproximadas: $1,0 \mathrm{~kg} / \mathrm{L}^{6}$.

Do ponto de vista estrutural, cada circunferência deve manter uma proporção matemática em relação à altura do indivíduo, conforme Figura 1 . 


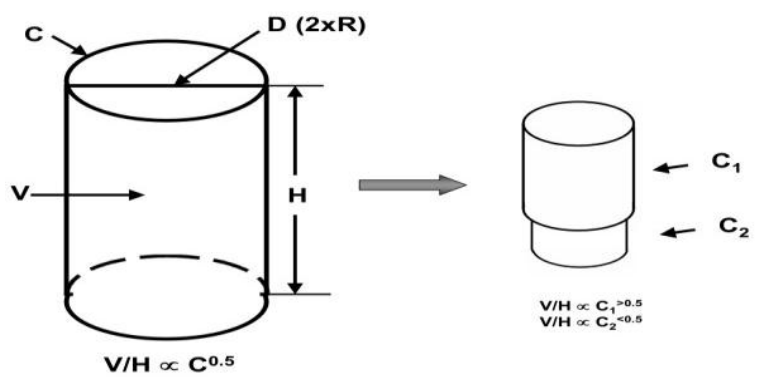

Figura 1: Modelo Hipotético de Cilíndrico Básico com Volume (à esquerda) e Modelo de Expansão com Volume de Dois Cilindros (HEYMSFIELD et al., 2008)

O raio do corpo (r) é equivalente ao comprimento da circunferência $2 \pi R$ (leia-se: 2 multiplicado por pi multiplicado pelo raio).

Assim, calcula-se o volume corporal em função das circunferências com base na Equação 1:

$$
\mathrm{V}=\pi .(\mathrm{C} / 2 \pi)^{2} . \mathrm{H}
$$

As circunferências se expressam pela Equação 2:

$$
\mathrm{C}=(4 \pi(\mathrm{V} / \mathrm{H}))^{0,5}
$$

Ainda, é possível correlacionar a Equação da 2 com o IMC através da substituição de V pelo peso corporal (W), constante na Equação 3:

$$
\mathrm{C} \alpha(\mathrm{V} / \mathrm{H})^{0,5}
$$

Assim, a Equação 4 resulta da fusão das duas equações acima, seja ela:

$$
\mathrm{C} \alpha(\mathrm{IMC} / \mathrm{H})^{0,5^{5}}
$$

\section{Metodologia}

Trata-se de um estudo transversal e comparativo, de abordagem quantitativa e qualitativa, tendo por objetivo a identificação do perfil demográfico e clínico de pacientes adultos portadores de cardiopatias, ou não, que realizam tratamento nos ambulatórios do HSJA, Itaperuna/RJ, a fim de correlacionar tais dados com as circunferências corporais e o risco de doença cardiovascular, adotados no NYORC (HEYMSFIELD et al., 2008).

A amostra foi obtida por conveniência, no período de agosto de 2014 a outubro de 2014, assim, foram recrutados $50 \%$ de indivíduos cardiopatas, incluídos no grupo G1, e 50\% de pacientes não cardiopatas, que compuseram o grupo G2. As investigações foram conduzidas sobrelevando as diferenças e semelhanças entre indivíduos cardiopatas (G1) e não cardiopatas $(\mathrm{G} 2)$.

Um total de 30 (trinta) pacientes foram admitidos à consulta cardiológica de rotina nos ambulatórios do HSJA. Os critérios de inclusão para G1 foram: (a) ter documentação de um episódio de IAM; (b) fazer uso contínuo de medicações para HAS, diabetes ou insuficiência cardíaca; (c) ter 18 (dezoito) anos ou mais; (d) prontuário cardiológico completo.

Similarmente, foram recrutados 30 (trinta) indivíduos em atendimento ambulatorial em diversas especialidades para compor G2. Os critérios de exclusão foram: (a) ter menos de 18 (dezoito) anos; (b) ausência de doença cardiovascular ou doenças crônicas doenças crônicas que possam afetar a função cardíaca; (c) não fazer uso de medicações anti-hipertensivas. 
Assim, os indivíduos foram divididos em dois grupos: (a) Grupo 1 (G1): pacientes cardiopatas; (b) grupo 2 (G2): pacientes não-cardiopatas.

As variáveis independentes foram obesidade, idade, peso (IMC $\geq 30 \mathrm{~kg} / \mathrm{m}^{2}$ ), circunferências e alturas do braço, cintura, quadril, coxa e panturrilha, renda mensal familiar, sedentarismo e tabagismo.

Os resultados serviram de base para a predição do risco, ou não, de maior risco para o desenvolvimento de doenças cardiovasculares.

Os indivíduos foram submetidos a medições através de balança mecânica com capacidade de $150 \mathrm{~kg}$ e a altura será aferida em metros (m).

As circunferências corporais foram medidas através de fita métrica com o sujeito amostral em posição ortostática e relaxada, tendo os braços pendentes ao longo do corpo.

O experimento abordou os cinco perímetros supracitados (HEYMSFIELD et al., 2008). O perímetro braquial medido de um ponto equidistante entre o acrômio da escápula direita e o olécrano da ulna. A circunferência da cintura foi medida em um ponto equidistante entre o rebordo costal até a crista ilíaca direita na linha axilar média. A circunferência do quadril foi medida 3 (três) centímetros abaixo da crista ilíaca, ao nível trocânter maior do fêmur e o ponto mais superior da borda lateral tíbia direita (ao nível da fossa poplítea). A panturrilha foi medida ao nível máximo da perna direita na face lateral com o sujeito em pé.

Todos os participantes da presente pesquisa assinaram o Termo de Consentimento Livre e Esclarecido, segundo a Resolução CNS 466/12.

Quanto à análise estatística, foram elaboradas tabelas descritivas, utilizando-se frequência, media e desvio-padrão. Além disso, o teste $t$ de Student serviu de instrumento para comparar as variações médias. Já para a correlação das frequências, utilizou-se o teste qui-quadrado de Pearson. As diferenças foram reputadas com nível de significância estatística fixada em $\mathrm{p}<0,05$.

\section{Resultados}

A idade média geral dos participantes da pesquisa foi de $47( \pm 13,34)$ anos.

É possível perceber que, nos pormenores da descrição, G1 exibiu médias dos resultados superiores ao G2 no que tange aos valores antropométricos (peso e altura), de obesidade (IMC), bem como às médias das circunferências corporais (braço, cintura e quadril) (Tabela 1). Ainda que, tanto em G1 quanto em G2, o IMC manteve-se acima dos valores preconizados como saudáveis $(25,82 \pm$ $4,55)$. 
Tabela 1: Dados Sociodemográficos.

\begin{tabular}{|c|c|c|c|}
\hline \multirow{3}{*}{ VARIÁVEIS } & \multicolumn{2}{|c|}{ GRUPOS } & \multirow[b]{2}{*}{ TOTAL } \\
\hline & G1 & G2 & \\
\hline & & & \\
\hline $\begin{array}{l}\text { Média } \pm \text { DP } \\
\text { Peso }(\mathrm{Kg})\end{array}$ & $49,2 \pm 12,85$ & $44,8 \pm 13,45$ & $47 \pm 13,34$ \\
\hline $\begin{array}{l}\text { Média } \pm \text { DP } \\
\text { Altura }(\mathrm{m})\end{array}$ & $73,83 \pm 14,96$ & $70,4 \pm 15,22$ & $72,11 \pm 15,19$ \\
\hline $\begin{array}{c}\text { Média } \pm \text { DP } \\
\text { IMC }\left(\mathrm{Kg} / \mathrm{m}^{2}\right)\end{array}$ & $1,67 \pm 0,89$ & $1,66 \pm 0,93$ & $1,67 \pm 0,91$ \\
\hline $\begin{array}{l}\text { Média } \pm \text { DP } \\
\text { Braço }(\mathrm{cm})\end{array}$ & $26,04 \pm 2,64$ & $25,38 \pm 5,66$ & $25,82 \pm 4,55$ \\
\hline $\begin{array}{c}\text { Média } \pm \text { DP } \\
\text { Cintura }(\mathrm{cm})\end{array}$ & $44,13 \pm 9,44$ & $38,33 \pm 9,00$ & $41,23 \pm 9,67$ \\
\hline $\begin{array}{l}\text { Média } \pm \text { DP } \\
\text { Quadril }(\mathrm{cm})\end{array}$ & $85,03 \pm 13,59$ & $81,83 \pm 11,16$ & $80,43 \pm 13,26$ \\
\hline $\begin{array}{c}\text { Média } \pm \text { DP } \\
\text { Coxa }(\mathrm{cm})\end{array}$ & $98,53 \pm 10,27$ & $95,33 \pm 10,73$ & $96,93 \pm 10,62$ \\
\hline $\begin{array}{c}\text { Média } \pm \text { DP } \\
\text { Panturrilha }(\mathrm{cm})\end{array}$ & $57,36 \pm 10,99$ & $61,37 \pm 13,06$ & $54,87 \pm 13,71$ \\
\hline Média \pm DP & $37,00 \pm 10,95$ & $43,70 \pm 13,00$ & $41,85 \pm 12,96$ \\
\hline
\end{tabular}

Com base em análise de regressão linear simples, demonstrou-se correlação fortemente significativa entre os índices de circunferência e volume/altura, de acordo com os coeficientes de correlação de Pearson (Tabela 2).

Tabela 2: Análise de Correlação entre Circunferência e Volume/Altura observada por regressão linear.

\begin{tabular}{ccc}
\hline INDICADORES & G1 & G2 \\
\hline Circunferência e & Coeficiente de & Coeficiente de \\
Volume/Altura & Pearson $(\boldsymbol{\rho})$ & Pearson $(\boldsymbol{\rho})$ \\
Braço & 0,991 & 0,996 \\
Cintura & 0,998 & 0,998 \\
Quadril & 0,999 & 0,999 \\
Coxa & 0,993 & 0,995 \\
Panturrilha & 0,995 & 0,993 \\
\hline
\end{tabular}

Conforme previsto no modelo geométrico desenvolvido pelo New York Obesity Research Center (NYORC) (HEYMSFIELD et al., 2008), as correlações entre as variáveis estudadas, devem fornecer medidas semelhantes. No entanto, as circunferências da cintura e do quadril ( $p=0,998$ e $\rho$ $=0,999$ ) apresentaram proporções iguais tanto em G1 quanto em G2.

$\mathrm{Na}$ comparação reiterativa dos dois grupos (Figura 2 e Figura 3), revela-se a correlação fortemente significativa da adiposidade da região abdominal (valores da circunferência da cintura e quadril, e volume/altura da cintura e do quadril) nos cardiopatas quanto nos não cardiopatas, o que pode ser explicado pelos indicadores de obesidade (IMC) acima dos valores recomendados (IMC > $25 \mathrm{~kg} / \mathrm{m}^{2}$ ) pela Sociedade Brasileira de Cardiologia (2014).

Embora o eixo Y de G2 (G2-Volume/Altura da cintura) apresente valores levemente menores que G1, a semelhança entre os dois grupos foi sustentada na análise regressão linear. 


\section{ACTA}
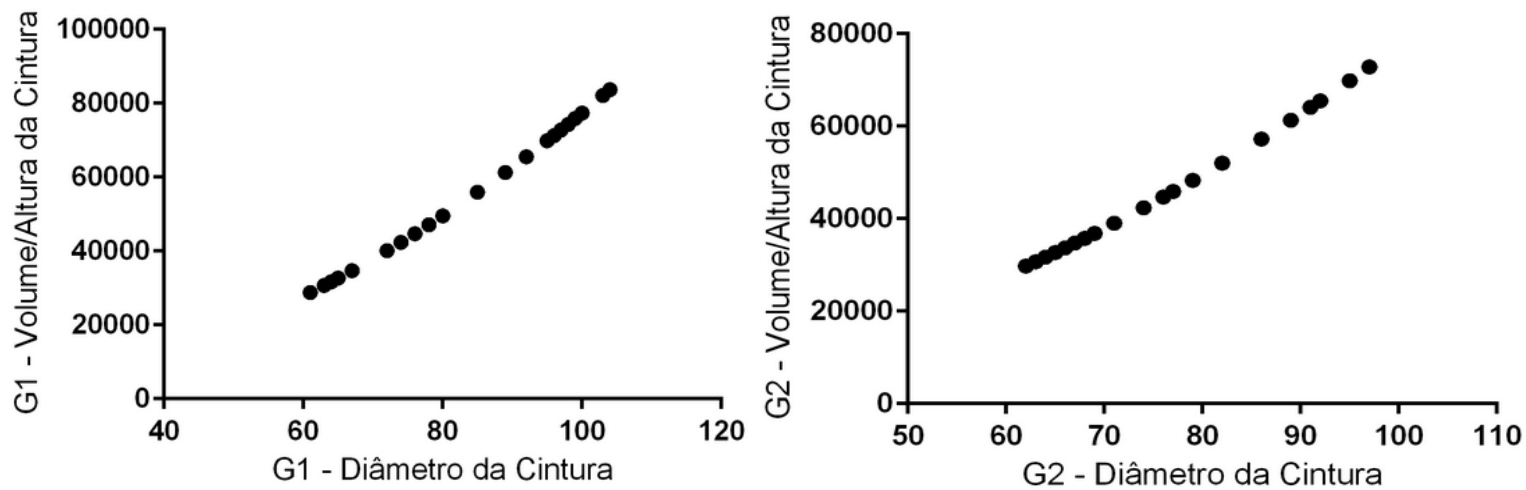

Figura 2: Regressão Linear entre Diâmetro e Volume/Altura da Cintura.
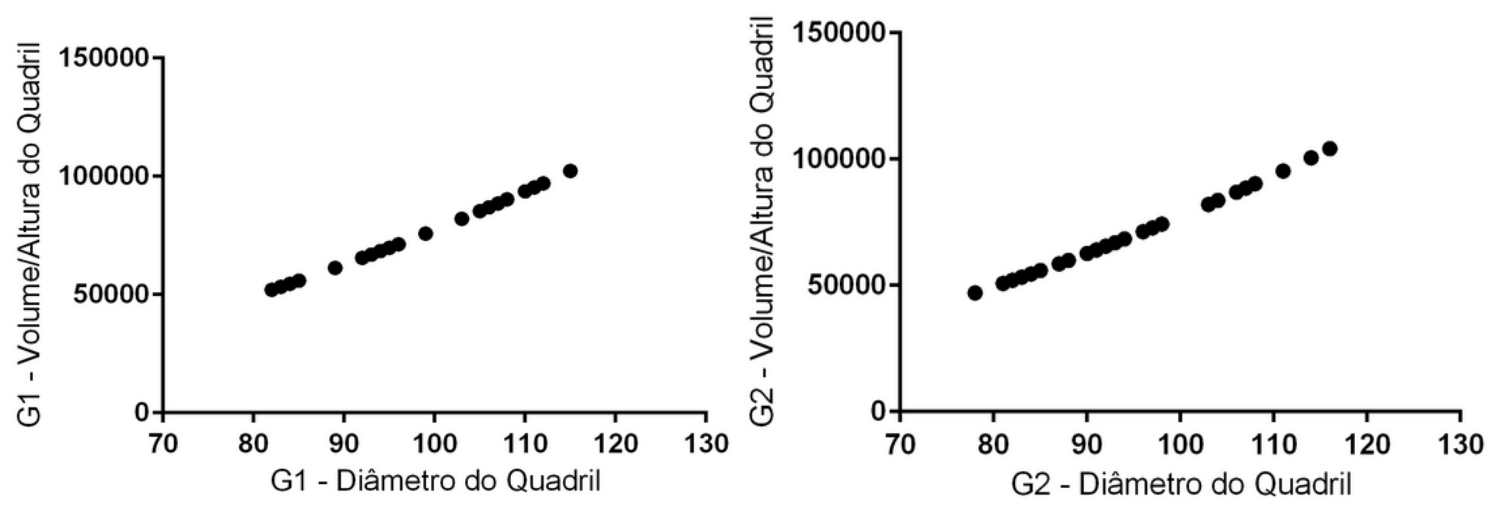

Figura 3: Regressão Linear entre Diâmetro e Volume/Altura do Quadril.

Os dados constantes nas Figuras 4 e 5 formam uma matriz quadrada de correlação, onde o número de colunas é igual ao número de linhas, com 1 (um) na diagonal. Portanto, os triângulos superiores direitos são o inverso dos triângulos inferiores esquerdos. Neles, A1, A2, A3, A4, A5, V1, V2, V3, V4 e V5 representam, respectivamente, a Altura e o Volume $\left(\mathrm{V}=\pi(\mathrm{C} / 2 \pi)^{2}\right.$. H), das 05 (cinco) circunferências estudadas, sejam elas, braço, cintura, quadril, coxa e panturrilha. Assim, reitera-se a correlação entre as variáveis através da álgebra linear aplicada.

\begin{tabular}{||c||c|c||c|c|c|c|c|c||c||c||}
\hline & V1 & V2 & V3 & V4 & V5 & V6 & V7 & V8 & V9 & V10 \\
\hline \hline V1 & 1 & 0.991 & 0.919 & 0.903 & 0.842 & 0.828 & 0.878 & 0.863 & 0.923 & 0.897 \\
\hline V2 & 0.991 & 1 & 0.888 & 0.875 & 0.81 & 0.798 & 0.872 & 0.865 & 0.893 & 0.873 \\
\hline V3 & 0.919 & 0.888 & 1 & 0.998 & 0.947 & 0.942 & 0.904 & 0.89 & 0.995 & 0.991 \\
\hline V4 & 0.903 & 0.875 & 0.998 & 1 & 0.949 & 0.946 & 0.905 & 0.895 & 0.992 & 0.993 \\
\hline V5 & 0.842 & 0.81 & 0.947 & 0.949 & 1 & 0.999 & 0.86 & 0.852 & 0.938 & 0.94 \\
\hline V6 & 0.828 & 0.798 & 0.942 & 0.946 & 0.999 & 1 & 0.858 & 0.852 & 0.932 & 0.937 \\
\hline V7 & 0.878 & 0.872 & 0.904 & 0.905 & 0.86 & 0.858 & 1 & 0.993 & 0.908 & 0.911 \\
\hline V8 & 0.863 & 0.865 & 0.89 & 0.895 & 0.852 & 0.852 & 0.993 & 1 & 0.894 & 0.904 \\
\hline V9 & 0.923 & 0.893 & 0.995 & 0.992 & 0.938 & 0.932 & 0.908 & 0.894 & 1 & 0.995 \\
\hline V10 & 0.897 & 0.873 & 0.991 & 0.993 & 0.94 & 0.937 & 0.911 & 0.904 & 0.995 & 1 \\
\hline
\end{tabular}

Figura 3: Matriz de Correlação das Circunferências pelos Volumes/Alturas (V/H) Corporais do G1. 


\begin{tabular}{|c||c|c|c|c|c||c|c||c||c||c||}
\hline & V1 & V2 & V3 & V4 & V5 & V6 & V7 & V8 & V9 & V10 \\
\hline \hline V1 & 1 & 0.997 & 0.972 & 0.958 & 0.904 & 0.901 & 0.948 & 0.93 & 0.962 & 0.932 \\
\hline \hline V2 & 0.997 & 1 & 0.977 & 0.967 & 0.912 & 0.912 & 0.952 & 0.941 & 0.967 & 0.945 \\
\hline V3 & 0.972 & 0.977 & 1 & 0.998 & 0.926 & 0.929 & 0.977 & 0.977 & 0.996 & 0.989 \\
\hline V4 & 0.958 & 0.967 & 0.998 & 1 & 0.924 & 0.929 & 0.976 & 0.981 & 0.995 & 0.994 \\
\hline V5 & 0.904 & 0.912 & 0.926 & 0.924 & 1 & 0.999 & 0.928 & 0.925 & 0.924 & 0.914 \\
\hline V6 & 0.901 & 0.912 & 0.929 & 0.929 & 0.999 & 1 & 0.93 & 0.93 & 0.927 & 0.921 \\
\hline V7 & 0.948 & 0.952 & 0.977 & 0.976 & 0.928 & 0.93 & 1 & 0.996 & 0.977 & 0.97 \\
\hline V8 & 0.93 & 0.941 & 0.977 & 0.981 & 0.925 & 0.93 & 0.996 & 1 & 0.978 & 0.981 \\
\hline \hline V9 & 0.962 & 0.967 & 0.996 & 0.995 & 0.924 & 0.927 & 0.977 & 0.978 & 1 & 0.993 \\
\hline V10 & 0.932 & 0.945 & 0.989 & 0.994 & 0.914 & 0.921 & 0.97 & 0.981 & 0.993 & 1 \\
\hline \hline
\end{tabular}

Figura 5: Matriz de Correlação das Circunferências pelos Volumes/Alturas (V/H) Corporais do G2.

\section{Discussão}

Diante do propósito do estudo, alguns parâmetros, ainda não citados, detalhadamente, foram consistentes com outros estudos. Sejam eles, a associação do risco cardiovascular com as circunferências corporais, com o IMC e o tabagismo.

Ao optar por realizar análise de regressão linear, para obtenção dos valores mais expressivos da relação V/H, através da correlação de Pearson, os estudiosos do NYORC (HEYMSFIELD et al., 2008), obtiveram maior significância para as medidas do braço (homens: $p=0,976$; mulheres: $\mathrm{p}=0,980$ ) e da cintura (homens: $\mathrm{p}=0,956$; mulheres: $\mathrm{p}=0,984$ ). O estudo brasileiro exibiu medidas semelhantes nos grupos estudados, no entanto, com destaque para a medida da cintura (G1: 0,998, G2: 0,998) e a do quadril (G1: 0,999, G2: 0,999).

Nesta pesquisa, ao evidenciar medidas exuberantes da cintura e do quadril nos grupos de estudo, fez-se jus, também, à importância do IMC como preditor de doenças coronarianas. De fato, houve prevalência de sobrepeso (IMC $>25 \mathrm{~kg} / \mathrm{m}^{2}$ ) tanto em G1 quanto em G2. G1 apresentou IMC com média de $26,04 \mathrm{~kg} / \mathrm{m}^{2}( \pm 2,64)$, estatura média de $1,67 \mathrm{~m}( \pm 0,89)$ e peso médio de $73,83 \mathrm{~kg} / \mathrm{m}^{2}$ $( \pm 14,96)$. Em G2, o IMC médio foi de $25,38 \mathrm{~kg} / \mathrm{m}^{2}( \pm 5,66)$, a estatura média de $1,66 \mathrm{~m}( \pm 0,93) \mathrm{e}$ peso médio de $70,4 \mathrm{~kg}( \pm 15,22)$.

Conforme apontado por Müezziener et al., ao analisar a mortalidade em 489.056 sujeitos tabagistas, os estudiosos perceberam que os índices de mortalidades eram duas vezes superiores em comparação com pessoas que nunca fumaram. Para perceber as nuanças da correlação entre tabagismo e doença cardiovascular, verificou-se que $60 \%(n=18)$ do grupo cardiopata $(\mathrm{G} 1)$ eram tabagistas, enquanto $40 \%(\mathrm{n}=12)$ dos não cardiopatas $(\mathrm{G} 2)$ eram tabagistas.

Segundo o estudo de Thijssen et al (2008), o sedentarismo foi um fator de risco para aterosclerose, doenças cardiovasculares e diabetes. Outrossim, a baixa aptidão cardiorrespiratória foi um fator preditor de mortalidade. Comparativamente, ao desvelar o sedentarismo, no estudo em tela, é preciso atentar ao fato de que, no $\mathrm{G} 1(\mathrm{n}=17,56 \%)$, o número de cardiopatas sedentários foi superior ao número de não cardiopatas sedentários em G2 $(\mathrm{n}=14,46 \%)$. 


\section{Conclusão}

A principal contribuição deste estudo é alertar os profissionais médicos sobre os deletérios da obesidade (BRASIL, 2001; CANOY, 2008; FARIA, 2013), além de ampliar os limites da prevenção da doença cardiovascular.

Os estudos do grupo de Framingham (HEITMANN, 2009) ampararam o deslocamento do foco do tratamento em pacientes com doenças cardiovasculares para a prevenção da patologia em pessoas com fatores de risco. Apoiados em vários matizes, foram identificados os fatores de risco metabólicos e cardiovasculares para o desenvolvimento da Insuficiência Cardíaca (IC). Assim, em princípio, a HAS, o diabetes mellitus, as dislipidemias, a obesidade, o tabagismo e o sedentarismo foram identificados como fatores de risco.

\section{Referências}

ABEL, E.D.; LITWIN, S.E.; SWEENEY, G. Cardiac Remodeling in Obesity. Physiol Rev. Boston: v. 88, n. $2,2008$.

BRASIL. Diretrizes Curriculares Nacionais do Curso de Graduação em Medicina. Resolução CNE/CES no 4, de 7 de novembro de 2001.

CANOY, D. Distribution of body fat and risk of coronary heart disease in men and women. Curr Opin Cardiol. Manchester: v. 23, n. 6, 2008.

FARIA, F.R. Body Fat Equations and Electrical Bioimpedance Values in Prediction of Cardiovascular Risk Factors in Eutrophic and Overweight Adolescents. Int J Endocrinol. v. 2.013, n. 501638, 2013.

HEITMANN, B.L. Thigh circumference and risk of heart disease and premature death: prospective cohort study. BMJ. Londres: v. 339, n. B3292, 2009.

Hip circumference and cardiovascular morbidity and mortality in men and women.

Obes Res. Londres: v. 12, n. 3, 2004.

HSIEH, S.D.; YOSHINAGA, H. Abdominal fat distribution and coronary heart disease risk factors in men-waist/height ratio as a simple and useful predictor. Int $\mathbf{J}$ Obes Relat Metab Disord. Londres: v. 19, n. 8, 1995.

HEYMSFIELD, S.B. et al. Body Circumferences: clinical implications emerging from a new geometric model. Nutr Metab. Londres: v. 24, n. 5, 2008.

JUNG, K.J.et al. Thigh Circumference and Diabetes: Obesity as a Potential Effect Modifier. Journal of Epidemiology. Oxford: v. 23, n. 5, 2013.

O'NEILL, T. et al. Combined Use of Waist and Hip Circumference to Identify Abdominally Obese HIV-Infected Patients at Increased Health Risk. PLoS ONE. v. 8, n.5, 2010. 
SOCIEDADE BRASILEIRA DE CARDIOLOGIA. IV Diretrizes Brasileiras sobre Dislipidemias e Prevenção de Aterosclerose. Disponível em: <http://www.prefeitura.sp.gov.br/cidade/secretarias/upload/saude/arquivos/programas/Diretriz_Bras ileira_Dislipidemias_Aterosclerose.pdf>. Acesso em: 07 de fevereiro de 2016.

SNIJDER, M.B. et al. Associations of hip and thigh circumferences independent of waist circumference with the incidence of type 2 diabetes: the Hoorn Study. J Clin Nutr. Amsterdã: v. 77 , n. 5, 2003.

THIJSSEN, D.H.J. et al. Physical (in)activity and endothelium-derived constricting factors: overlook adaptations. J Physiol. Amsterdã: v. 586, n. 2, 2008. 